\title{
Gut flora - pathogenic role in chronic heart failure
}

Chronic heart failure is associated with a huge increase in levels of pathogenic bacteria and fungi in the gut, as well as increases in intestinal permeability, right atrial pressure, and inflammation. These findings come from a study conducted in Italy and now published in JACC Heart Failure.

Chronic systemic inflammation is known to occur in patients with heart failure, and this inflammatory state has been associated with alterations in the morphology, function, and bacterial flora of the intestine. Flourishing of bacterial populations coupled with increased gut permeability might result in bacteria and their toxins entering the bloodstream and causing inflammation. Accordingly, Evasio Pasini and colleagues set out to identify and quantify the colonies of pathogenic bacteria in the gut, and to determine whether there was a correlation with the severity of heart failure.

The investigators recruited 60 well-nourished, stable patients with chronic heart failure, 30 of whom were in NYHA class I-II (classified as mild heart failure) and 30 in NYHA class III-IV (moderate-to-severe heart failure). They also enrolled 20 matched, healthy control individuals. Participants had not undergone any antibiotic, steroid, antidiarrhoeal, or probiotic treatment in the past 3 months. Gut flora was determined by the development of bacteria and fungi (Candida species) in faeces. Disaccharide cellobiose content in the urine after a sugar drink test was quantified to evaluate intestinal permeability. Right atrial pressure was assessed using echocardiography, and the level of C-reactive protein was measured as a marker of systemic inflammation.

Compared with healthy individuals, patients with chronic heart failure had more colonies of pathogenic bacteria, including species of Campylobacter, Salmonella, Shigella, and Yersinia enterocolitica. Candida species were also present. Compared with patients with mild heart failure, more patients with moderate-tosevere heart failure had Candida, Campylobacter, and Shigella species in their stools.

Intestinal permeability was normal in healthy controls ( $1.5 \pm 0.8 \mathrm{mg})$, but was increased in $78.5 \%$ of patients with heart failure (10.2 \pm 1.2 mg; $P<0.001$ ). The intestine was more permeable in patients with moderate-to-severe heart failure $(12.4 \pm 1.1 \mathrm{mg})$ than in those with mild heart failure $(7.3 \pm 0.9 \mathrm{mg})$. Right atrial pressure was higher in patients with moderate-to-severe heart failure $(12.6 \pm 0.6 \mathrm{mmHg})$ than in patients with mild heart failure $(6.3 \pm 0.4 \mathrm{mmHg})$ and control individuals $(0.2 \pm 0.08 \mathrm{mmHg})$. Similarly, levels of C-reactive protein were elevated in patients with moderate-to-severe heart failure $(12.5 \pm 0.6 \mathrm{mg} / \mathrm{dl})$

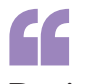

Patients with chronic heart failure had more colonies of pathogenic bacteria

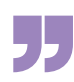

and, to a lesser extent, in those with mild heart failure $(6.0 \pm 0.3 \mathrm{mg} / \mathrm{dl})$, but was in the normal range in healthy controls $(0.15 \pm 0.02 \mathrm{mg} / \mathrm{dl})$. Statistical analysis showed that intestinal permeability, right atrial pressure, and the level of C-reactive protein were mutually interrelated.

The investigators conclude that "patients with chronic heart failure had intestinal overgrowth of pathogenic bacteria and increased intestinal permeability. Moreover, the results show that intestinal permeability was associated with inflammation, right atrial pressure, and clinical disease severity." The researchers believe that "the pathogenic gut flora is probably a major factor of intestinal permeability because these microorganisms can cause chronic intestinal wall and systemic inflammation".

Given that pathogenic gut flora and increased intestinal permeability can complicate the clinical course of patients with heart failure, the investigators suggest that "gut microbiota should be continually investigated as soon as chronic heart failure is diagnosed". At present, however, no clinical modifiers of gut flora are available, and some evidence indicates that the use of probiotics might be dangerous.

Gregory B. Lim 